\title{
Minor segmental wall motion abnormalities detected in patients with Chagas' disease have adverse prognostic implications
}

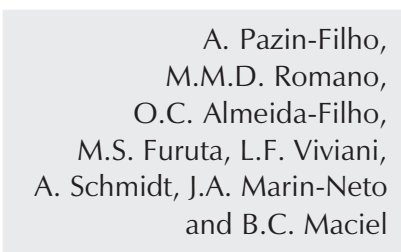

\author{
Divisão de Cardiologia, Departamento de Clínica Médica, \\ Faculdade de Medicina de Ribeirão Preto, Universidade de São Paulo, \\ Ribeirão Preto, SP, Brasil
}

\section{Correspondence \\ B.C. Maciel \\ Divisão de Cardiologia \\ Departamento de Clínica Médica \\ FMRP, USP \\ Av. Bandeirantes, 3900 \\ 14048-900 Ribeirão Preto, SP \\ Brasil \\ Fax: +55-16-3633-0869 \\ E-mail: bcmaciel@fmrp.usp.br}

Publication supported by FAPESP. ..............

Received May 18, 2005 Accepted January 2, 2006

\begin{abstract}
Recent data from our laboratory have shown that patients with the indeterminate form of Chagas' disease can have impairment of left ventricular contractility, as evaluated by the slope of the left ventricle end-systolic pressure-dimension relationship. We also showed that Chagas' disease patients with minimal baseline wall motion abnormalities detected by two-dimensional echocardiography have more intense contractility impairment when compared to patients with the indeterminate form of the disease without this abnormality. The prognostic implications of these findings have not been established. We evaluated 59 patients (37-76 years, mean $=55$ years) with different clinical forms of Chagas' disease, who had normal left ventricular global systolic function at baseline $(57.6 \pm 6.9 \%)$ and who had at least one additional echo during clinical follow-up (0.4-17.6; mean 4.6 years). Group 1 consisted of 14 patients with minor baseline left ventricle wall motion abnormalities and group 2 consisted of 45 patients without these abnormalities. During follow-up, global left ventricle systolic function deterioration was observed in 10 group 1 patients $(71.4 \%)$ and in only 10 group 2 patients $(22.2 \%$; $\mathrm{P}<0.005)$. Age and duration of follow-up were not independent determinants of left ventricular function deterioration in these patients. The present data indicate that mild segmental left ventricular wall motion abnormalities are associated with worsening of systolic function in Chagas' disease patients who have normal baseline global systolic performance.
\end{abstract}

Chagas' disease remains a significant public health issue in many countries and is a major cause of morbidity and mortality in Latin America. The social impact of this disease can be evaluated by the estimated human infection prevalence of 16 million
Key words

- Echocardiography

- Chagas' disease

- Left ventricular function

- Indeterminate form of

Chagas' disease people (1) in this region. Chronic Chagas' heart disease is certainly the most important clinical form of Chagas' disease. Chronic cardiac involvement is more prevalent and is also associated with grater morbidity and mortality when compared to the digestive 
form $(2,3)$. In the natural history of the disease, the progression of cardiac involvement is heterogeneous and tends to be slow: about $70 \%$ of cardiac patients who have mild cardiac involvement, without heart failure, remain clinically stable for periods ranging from 12 to 27 years of follow-up (4).

The majority of patients with Chagas' disease remain in the indeterminate form of the disease for 10-30 years or even more. They have positive serology, no symptoms or signs of Chagas' disease, and normal ECG and heart, esophagus and colon X-rays (5). Different diagnostic techniques (vectorcardiography, echocardiography, radionuclide ventriculography, exercise stress test, Holter monitoring, endomyocardial biopsy) have shown some degree of cardiac abnormalities in a substantial number of these patients (5). Although their prognostic implications are not completely clear, these findings do not appear to significantly influence the progression of the disease to symptomatic clinical forms, and patients with the indeterminate form usually have an excellent long-term prognosis. Despite the discrepant observations regarding ventricular systolic dysfunction in patients with the indeterminate form of Chagas' disease, it should be pointed out that a substantial proportion of these patients may have minor impairment of segmental ventricular function. We have previously shown (6) that patients with the indeterminate form of Chagas' disease can have impairment of left ventricular contractility, as evaluated by the slope of the left ventricle end-systolic pressure-dimension relationship. Our results also showed that, independent of the clinical classification, patients who had minor left ventricular segmental wall motion abnormalities (LVWMA) on baseline two-dimensional (2-D) echocardiogram presented a more depressed myocardial contractility when compared to patients who had only isolated conduction abnormalities (6) on ECG. These data were consistent with a more extensive and diffuse myocardial involvement in these patients. Although the clinical classification of patients with Chagas' disease may vary from country to country (7), in Brazil, mild isolated regional wall motion abnormalities documented in Chagas' disease patients are not unanimously considered to be a hallmark for classifying a patient as presenting the cardiac form of the disease.

To investigate whether minor LVWMA documented on baseline 2-D echocardiography in Chagas' disease patients is associated with a worsening of ventricular systolic function during long-term follow-up, we evaluated patients who fulfilled the following inclusion criteria: 1) different clinical forms of Chagas' disease but with preserved global left ventricular systolic function; 2) at least two echocardiographic examinations during follow-up; 3) no other associated diseases. Clinical classification of patients with Chagas' disease was based on the following criteria: 1) indeterminate form: patients had a positive serum reaction for Chagas' disease, no digestive or cardiac symptoms, normal resting electrocardiogram and a normal esophageal X-ray; 2) digestive form: patients had symptoms of dysphagia or constipation and an abnormal esophageal or colon X-ray; 3) cardiac form: patients presenting right bundle branch block and/or left anterior fascicular block, but no cardiorespiratory symptoms and no enlargement of ventricular chambers on 2-D echocardiography and chest X-ray. In addition to these traditional criteria, we have also used a different classification considering the cardiac form to be present in all patients who had mild left ventricular systolic segmental dysfunction but normal left ventricular global function on 2-D echocardiography. Therefore, in this new classification, to be considered in the indeterminate or digestive form, patients must present normal left ventricular segmental wall motion on 2D echocardiography. All subjects gave written informed consent to participate in this study and the protocol was approved by the 
University Hospital Research Ethics Committee.

Fifty-nine patients (mean age 55.1 years; range: 37-76 years) who fulfilled the inclusion criteria were selected based on the traditional clinical classification, 23 had the cardiac form (38\%), 17 the digestive form $(30 \%)$ and 19 the undetermined form $(32 \%)$; using the new classification 30 had the cardiac form $(51 \%), 13$ the digestive form (22\%) and 16 had the undetermined form (27\%). The mean average follow-up was 4.6 years (range: 0.4-17.6 years). The patients were divided into two groups: 1) with LVWMA $(\mathrm{N}=14)$ and 2) without LVWMA, at baseline 2-D echocardiography $(\mathrm{N}=45)$. The two groups were analyzed to identify any worsening of left ventricular systolic function (extension of LVWMA as documented by worsening of wall motion in at least two left ventricular segments based on semiquantitative grading and/or reduction of at least $5 \%$ in left ventricular ejection fraction) during follow-up. 2-D left ventricular ejection fraction was obtained off-line using the Simpson's method, incorporated into the ultrasound system.

Data are reported as mean $\pm \mathrm{SD}$. The chisquare test was used to compare proportions of patients with or without left ventricular deterioration during follow-up. A P value < 0.05 was considered to be statistically significant.

Baseline mean ejection fraction was 57.6 $\pm 6.9 \%$. Among patients presenting minor LVWMA, 71.4\% showed worsening of left ventricular systolic function during followup while only $22.2 \%$ of those without LVWMA had left ventricular systolic function deterioration $(\mathrm{P}=0.004$; chi-square test; Table 1). The number of patients with different clinical forms of Chagas' disease who had worsening of left ventricular wall motion on 2-D echocardiography during follow-up is shown in Table 2. The deterioration of left ventricular systolic function was not dependent on patient age or duration of follow-up (chi-square test).

The clinical manifestations of the chronic cardiac phase of Chagas' disease are quite variable: patients can be asymptomatic or may present different types of arrhythmias (including potentially lethal arrhythmias), atrioventricular block, conduction abnormali-

\begin{tabular}{|c|c|c|c|}
\hline & \multicolumn{2}{|c|}{$\begin{array}{c}\text { Deterioration of } \\
\text { left ventricle systolic } \\
\text { function during follow-up }\end{array}$} & \multirow[t]{2}{*}{ Total } \\
\hline & Yes & No & \\
\hline \multicolumn{4}{|c|}{ Baseline LVWMA } \\
\hline Present & 10 & 4 & 14 \\
\hline Absent & 10 & 35 & 45 \\
\hline Total & 20 & 39 & 59 \\
\hline
\end{tabular}

Data are reported as number of patients.

Table 2. Deterioration of left ventricular systolic function during follow-up of the different clinical forms of Chagas' disease according to the traditional and new classification systems.

\begin{tabular}{|c|c|c|c|c|c|c|c|c|}
\hline & \multicolumn{4}{|c|}{ Traditional classification } & \multicolumn{4}{|c|}{ New classification } \\
\hline & $\begin{array}{l}\text { Indeter- } \\
\text { minate }\end{array}$ & Digestive & Cardiac & Total & $\begin{array}{l}\text { Indeter- } \\
\text { minate }\end{array}$ & Digestive & Cardiac & Total \\
\hline Baseline LVWMA + & $3(1)$ & $4(3)$ & $7(6)$ & $14(10)$ & - & - & $14(10)$ & $14(10)$ \\
\hline Baseline LVWMA - & $16(2)$ & $13(1)$ & $16(7)$ & $45(10)$ & $16(2)$ & $13(1)$ & $16(7)$ & $45(10)$ \\
\hline Total & $19(3)$ & $17(4)$ & $23(13)$ & $59(20)$ & $16(2)$ & $13(1)$ & $30(17)$ & $59(20)$ \\
\hline
\end{tabular}

The number of patients who had worsening of left ventricular systolic function is reported in parentheses. LVWMA = left ventricular wall motion abnormalities; +/- = presence or absence of LVWMA. 
ties on electrocardiogram, arterial or pulmonary embolism, or congestive heart failure (4). Left ventricular dysfunction, left ventricular aneurysm and congestive heart failure are recognized as important predictors of mortality in these patients. The magnitude of changes in left ventricular systolic function in Chagas' disease is quite variable depending on the degree of myocardial involvement of each patient. Segmental LVWMA based on echocardiography and radionuclide or contrast ventriculography has shown a number of abnormalities in chronic cardiac chagasic patients: apical dyskinesia was documented in $50-65 \%$ of symptomatic cardiac patients, while akinesia or hypokinesia was observed in the same region in $20 \%$ of patients; on the other hand, wall motion abnormalities involving the postero-inferior wall are reported in 15-20\% of patients and are rarely detected in the anterolateral wall; finally, diffuse hypokinesia was observed in $15-40 \%$ of patients (8). A significant proportion of these patients have segmental wall motion abnormalities documented in more than one left ventricular region. It is especially relevant to mention that this variable pattern of segmental involvement of the left ventricle is not related to obstructive lesions in epicardial coronary arteries, which are usually normal or even dilated in chagasic patients (4).

Evaluation of left ventricular systolic function in patients with the indeterminate form of Chagas' disease has shown discrepant results ranging from absence of any ventricular function abnormality to the occurrence of apical dyskinesia or akinesia in 10$68 \%$ of patients (8). Despite these heterogeneous observations regarding the degree of myocardial involvement in the indeterminate form of the disease, and even considering the limitation related to inadequate clinical characterization of the indeterminate form in several investigations (5), it should be recognized that a substantial proportion of these patients have minor impairment of segmental ventricular function (9).

The present findings indicate that minor LVWMA documented at baseline 2-D echocardiography in Chagas' disease patients who have normal global systolic function is a predictor of deterioration of ventricular function during follow-up. In addition, the present results suggest that patients who fulfill current criteria for the indeterminate form of Chagas' disease (no symptoms or physical signs, specific serological positivity, normal ECG and heart, esophagus and colon Xrays) but have minor LVWMA in 2-D echocardiography should be considered to present the cardiac form of Chagas' disease. The present findings also support the proposal that an objective assessment of left ventricle function should always be carried out in Chagas' disease patients presenting the indeterminate form of the disease (8). We believe that this preliminary observation should be confirmed in a larger prospective study.

Considering that angiotensin converting enzyme inhibitors and B-blockers are currently recommended for patients with cardiac structural abnormalities of variable etiologies who have not developed heart failure (10), it is a reasonable hypothesis that angiotensin converting enzyme inhibitors and ß-blockers may prevent further deterioration of ventricular function in these chagasic patients. 


\section{References}

1. Wanderley DMV \& Corrêa FMA (1995). Epidemiology of Chagas' heart disease. São Paulo Medical Journal, 113: 742-749.

2. Coura JR (1988). Determinantes epidemiológicos da doença de Chagas no Brasil: a infecção, a doença e sua morbi-mortalidade. Memórias do Instituto Oswaldo Cruz, 83 (Suppl 1): 392-402.

3. Dias JCP (1985). História natural. In: Cançado JR \& Chuster M (Editores), Cardiopatia Chagásica. Fundação Carlos Chagas, Belo Horizonte, MG, Brazil.

4. Marin-Neto JA, Simões MV \& Maciel BC (1998). Other cardiomyopathies. In: Yusif S, Cairns JA, Camin AJ et al. (Editors), Evidence Based Cardiology. BMJ Books, London, UK.

5. Pereira-Barreto AC \& lanni BM (1995). The undetermined form of Chagas' heart disease: concept and forensic implications. São Paulo Medical Journal, 113: 797-801.

6. Almeida-Filho OC, Maciel BC, Schmidt A et al. (2002). Minor seg- mental dyssynergy reflects extensive myocardial damage and global left ventricle dysfunction in chronic Chagas disease. Journal of the American Society of Echocardiography, 15: 610-616.

7. Hagar JM \& Rahimtoola SH (1995). Chagas' heart disease. Current Problems in Cardiology, 20: 825-924.

8. Maciel BC, Almeida-Filho OC, Schmidt A et al. (1995). Ventricular function in Chagas' heart disease. São Paulo Medical Journal, 113: 814-820.

9. Marin-Neto JA, Pazin-Filho A, Schmidt A et al. (2002). Forma indeterminada da moléstia de Chagas. Proposta de novos critérios de caracterização e perspectivas de tratamento precoce da cardiomiopatia. Arquivos Brasileiros de Cardiologia, 79: 623-627.

10. ACC/AHA (2005). Guideline update for the diagnosis and management of chronic heart failure in the adult. Journal of the American College of Cardiology, 46: 1116-1143. 